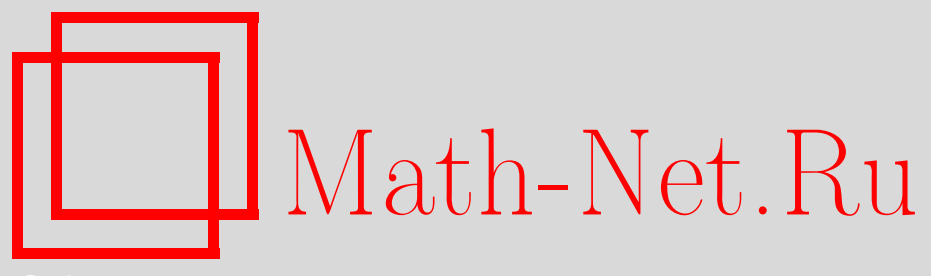

М. А. Комаров, Локальная управляемость в типичных двупараметрических семействах бидинамических систем на плоскости, УМН, 2008, том 63, выпуск 2, 169 170

DOI: https://doi.org/10.4213/rm9173

Использование Общероссийского математического портала Math-Net.Ru подразумевает, что вы прочитали и согласны с пользовательским соглашением http://www.mathnet.ru/rus/agreement

Параметры загрузки:

IP : 107.22 .136 .117

26 апреля 2023 г., 10:57:05

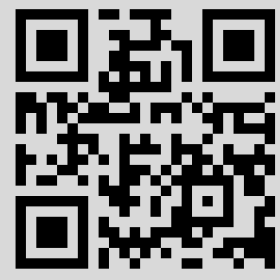




\title{
Локальная управляемость в типичных двупараметрических семействах бидинамических систем на плоскости
}

\author{
М. А. Комаров
}

Полидинамическая система на гладком (класса $C^{\infty}$ ) многообразии задается конечным набором гладких полей скоростей. Управление такой системой представляет собой выбор в последовательные моменты времени одного из полей для дальнейшего движения.

Полидинамическая система называется локально управляемой в точке $P$ многообразия, если существует время $T>0$ такое, что для любой окрестности $U$ этой точки найдется меньшая окрестность $V \subset U$, через каждую точку $p$ которой можно провести кусочно гладкую замкнутую кривую (цикл), целиком лежащую в $U$, проходящую через $P$ и состоящую из отрезков фазовых кривых полей системы, соответствующих некоторому управлению, причем время движения по циклу меньше $T$.

Для плоскости случаи локальной управляемости типичных полидинамических систем и бидинамических систем типичного однопараметрического семейства были изучены в [1] и [2] соответственно (типичными мы называем объекты из некоторого открытого всюду плотного множества в пространстве объектов, снабженном тонкой гладкой топологией, а бидинамической - полидинамическую систему с двумя полями скоростей). В настоящей работе представлены все возможные случаи локальной управляемости бидинамических систем типичного двупараметрического семейства на плоскости.

Понятно, что локальная управляемость сохраняется при переходе к семейству, гладко эквивалентному данному, т. е. получаемому диффеоморфизмами фазового пространства, гладко зависящими от параметров, и диффеоморфизмами пространства параметров. Поскольку в точке с локальной управляемостью одно из полей обязательно ненулевое, анализ случаев сводится к изучению семейства вида

$$
\left\{v=(0,1) ; w=\left(w_{1}, w_{2}\right)\right\},
$$

в котором первое поле выпрямлено [3] вдоль второй координаты. Далее, известно, что если в некоторой точке оба поля ненулевые и фазовая кривая одного касается фазовой кривой другого с конечным порядком, то локальная управляемость в этой точке есть тогда и только тогда, когда поля в ней противоположно направлены, а порядок касания их фазовых кривых нечетный (для типичного двупараметрического семейства этот порядок не превосходит 4). Таким образом, остается изучить локальную управляемость систем типичного семейства (1) в точке, особой для второго поля. Обозначим через $D(w)$ дискриминант характеристического многочлена матрицы $w_{*}$ линеаризации поля $w$ в этой точке.

Теорема. В особой точке поля $w$ система из типичного двупараметрического семейства (1) обладает локальной управляемостью, если и только если или

F) $D(w)<0$, uлu

L) семейство (1) в этой точке гладко эквивалентно семейству $\left\{(0,1) ;\left(A y, w_{2}\right)\right\}$ в нуле, где $A(0,0) \neq 0, d w_{2}(0,0)=0,\left(w_{2}\right)_{x x}(0,0)<0$,

и не обладает локальной управляемостью, если и только если или

NF) $D(w)>0$, или

NJ) $w_{*}$ определяет жорданов узел, или

NL) семейство (1) в этой точке гладко эквивалентно семейству $\left\{(0,1) ;\left(A y, w_{2}\right)\right\}$ в нуле, где $A(0,0) \neq 0, d w_{2}(0,0)=0,\left(w_{2}\right)_{x x}(0,0)>0$.

Работа выполнена при поддержке РФФИ (грант № 06-01-00661a). 
ЗАмечАния. 1. Обозначения F) и L) мотивированы тем, что линеаризация второго поля системы в случае F) определяет фокус или центр, а в случае L) - расслоение плоскости на параллельные прямые. Случаи L) и NL), равно как и случай одновременного обращения полей в нуль, не встречаются у систем типичного однопараметрического семейства.

2. Перечисленные в теореме случаи исключают друг друга и являются инвариантами семейства (1) относительно гладкой эквивалентности. Они связаны, хотя и опосредованно, с инвариантами семейства двумерных аффинных управляемых систем с одним входом и неограниченным управлением, полученными (для одномерного параметра) в работах [4] и [5].

Схема доказательства теоремы представлена ниже в определителе случаев со следующими обозначениями: $P$ - изучаемая точка, которую мы принимаем за начало координат; $\Rightarrow$ - "следует"; $\mapsto n-$ переход на позицию $n$; $n-$ переход на позицию $n$, сопровождаемый гладким преобразованием семейства, сохраняющим первое поле выпрямленным.

1) $w(P)=0 \Rightarrow$ одно из двух: $\left(w_{1}\right)_{y}(P) \neq 0 \rightsquigarrow 2$, $\left(w_{1}\right)_{y}(P)=0 \mapsto 10$

2) $w=\left[A(x, y, \varepsilon) y, w_{2}\right]$ с $A(P) \neq 0 \Rightarrow$ одно из двух: $\left(w_{2}\right)_{y}(P) \neq 0 \mapsto 3$, $\left(w_{2}\right)_{y}(P)=0 \mapsto 6 ;$

3) $w=[A(x, y, \varepsilon) y, B(x, y, \varepsilon)(y+b(x, \varepsilon))]$ c $(A B)(P) \neq 0=b(P) \mapsto 4$;

4) одно из двух: $D(w)=0 \Rightarrow(\mathrm{NJ})$, $D(w) \neq 0 \mapsto 5 ;$

5) одно из двух: $D(w)<0 \Rightarrow(\mathrm{F})$, $D(w)>0 \Rightarrow(\mathrm{NF})$

6) одно из двух: $\left(w_{2}\right)_{x}(P) \neq 0 \mapsto 7$, $\left(w_{2}\right)_{x}(P)=0 \Rightarrow\left(w_{2}\right)_{x x}(P) \neq 0 \rightsquigarrow 8 ;$

7) $w=[A(x, y, \varepsilon) y, B(x, y, \varepsilon)(x+b(y, \varepsilon))]$ c $(A B)(P) \neq 0=b(P)=b_{y}(P) \mapsto 5$;

8) $w=\left[A(x, y, \varepsilon) y, B(x, y, \varepsilon)\left(y^{2}+a(x, \varepsilon) y \pm x^{2}+\varepsilon_{1}\right)\right]$ с $a(P)=0 \neq(A B)(P) \mapsto 9$;

9) одно из двух: $\left(w_{2}\right)_{x x}(P)<0 \Rightarrow(\mathrm{L})$, $\left(w_{2}\right)_{x x}(P)>0 \Rightarrow(\mathrm{NL})$

10) одно из двух: $\left(w_{1}\right)_{x}(P) \neq 0 \mapsto 11$, $\left(w_{1}\right)_{x}(P)=0 \Rightarrow\left(w_{1}\right)_{y y}(P) \neq 0 \rightsquigarrow 14$

11) $w=\left[A(x, y, \varepsilon)(x+a(y, \varepsilon)), w_{2}\right]$ с $A(P) \neq 0=a_{y}(P) \Rightarrow$ одно из двух: $\left(w_{2}\right)_{y}(P) \neq 0 \mapsto 12$, $\left(w_{2}\right)_{y}(P)=0 \neq\left(w_{2}\right)_{x}(P) \Rightarrow(\mathrm{NF})$

12) $w=[A(x, y, \varepsilon)(x+a(y, \varepsilon)), B(x, y, \varepsilon)(y+b(x, \varepsilon))]$ с $(A B)(P) \neq 0=b(P) \Rightarrow$ одно из двух:

$$
\begin{aligned}
& A(P) \neq B(P) \Rightarrow(\mathrm{NF}), \\
& A(P)=B(P) \Rightarrow\left(w_{2}\right)_{x}(P) \neq 0 \rightsquigarrow 13 ;
\end{aligned}
$$

13) $w=[A(x, y, \varepsilon)(x+a(y, \varepsilon)), B(x, y, \varepsilon)(y+c(x, \varepsilon) x)] \mathrm{c}(A B c)(P) \neq 0=a(P)=$ $a_{y}(P) \Rightarrow(\mathrm{NJ})$

14) $w=\left[A(x, y, \varepsilon)\left(y^{2}+g(x, \varepsilon)\right), w_{2}\right]$ c $g(P)=g_{x}(P)=0 \neq\left(A\left(w_{2}\right)_{y}\right)(P) \Rightarrow(\mathrm{NF})$.

\section{Список литературы}

[1] A. A. Davydov, Qualitative theory of control systems, Transl. Math. Monogr., 141, Amer. Math. Soc., Providence, RI, 1994. [2] L. Azevedo, Transitividade local de sistemas polidinamicos, Master Thesis, Departamento de Matemática Aplicada, Faculdade de Ciências da Universidade do Porto, 2006. [3] В.И. Арнольд, Обыкновенные дифференииальные уравнения, 4-е изд., РХД, Ижевск, 2000. [4] В. Jakubczyk, W. Respondek, SIAM J. Control Optim., 44:6 (2006), 2038-2062. [5] M. W. Rupniewski, J. Dyn. Control Syst., 13:1 (2007), 135-159.

M. А. Комаров (M. А. Komarov)

Владимирский государственный университет

E-mail: kami9@yandex.ru
Представлено В. М. Закалюкиным Принято редколлегией 24.01.2008 